\title{
Pro und Kontra: „Jeder Schizophreniekranke sollte so früh wie möglich behandelt werden“"
}

\author{
For and Against: „Every Person with Schizophrenia Should be Treated \\ as Early as Possible“
}

\section{Pro}

Joachim Klosterkötter

Die Weltgesundheitsorganisation (WHO) hat aus guten Gründen die Prävention mentaler Störungen zu einer ihrer primären Zielsetzungen für die nächsten Jahre und Jahrzehnte erklärt. Ihr jüngster diesbezüglicher Bericht „Prevention of Mental Disorders - Effective Interventions and Policy Options“ listet sieben Störungsgruppen auf, für die sich heute schon Erfolg versprechende Präventionsprogramme umreißen lassen, und führt darunter auch die psychotischen Störungen an [1].

Wie könnte eine solche Programmatik speziell für die Schizophrenien tatsächlich aussehen? Eine universale Prävention, mit der man sich ohne Selektion einfach an die Allgemeinbevölkerung wenden und beispielsweise generelle Verbesserungen der Schwangerschafts- und Geburtsvorsorge anbieten könnte, käme wegen der relativ geringen Bedeutung der damit erreichbaren Krankheitsursachen, etwa der Geburtskomplikationen, nicht in Betracht. Auch eine selektive Prävention, bei der man sich stattdessen auf Bevölkerungsanteile mit erhöhtem Erkrankungsrisiko, etwa in Zukunft identifizierbare Genträger bezöge, würde sich wegen der nur geringen Vorhersagekraft aller bislang bekannten Risikofaktoren für Schizophrenie noch gar nicht verwirklichen lassen. Wenn jedoch zusätzlich zu solchen Risikofaktoren oder auch allein bestimmte Prodromalsymptome auftreten, bei deren Nachweis man einen Psychoseausbruch schon für die Folgejahre mit hoher Treffsicherheit vorhersagen kann, bietet sich schon eher ein Erfolg versprechender Ansatzpunkt für Prävention. Solche Frühwarnzeichen bewirken zudem oft selber schon quälenden Beschwerdedruck und sozial sehr hinderliche Funktionseinbußen, sodass die Betroffenen nach Rat und Hilfe suchen und ein gezieltes Interventionsangebot voll indiziert sein könnte. Nur diesen in der angloamerikanischen Versorgungsforschungund Gesundheitspolitik schon seit langem fest verankerten Ansatz der indizierten Prävention hat der WHO-Bericht im Blick, wenn er die Schizophrenie mit zu den bereits angehbaren psy- chischen Störungen zählt. Da auch bei den anderen genannten Gruppen, etwa den depressiven Störungen, Effektivitätsnachweise ganz überwiegend nur für diesen Ansatz vorliegen, täte man sicher gut daran, auch in Deutschland das Konzept der indizierten Prävention viel stärker zur Kenntnis zu nehmen als bisher und darin den ersten gangbaren Schritt auf dem Wege zu einer präventiven Psychiatrie zu sehen.

In den wichtigsten der seit den 90er-Jahren weltweit entstandenen Früherkennungszentren [2,3], die sich vorrangig auf psychotische Störungen beziehen, wird intensive Öffentlichkeitsarbeit betrieben, um Aufmerksamkeit für Risikofaktoren und Frühwarnzeichen sowie die entsprechenden Hilfsangebote zu erzeugen. Kommen Kontaktaufnahmen zustande, gilt es im Zuge subtiler Frühdiagnostik vor allem zu unterscheiden, ob sich die Betroffenen schon in der ersten psychotischen Episode, in einem bereits psychosenahen oder einem noch psychoseferneren Risikozustand befinden. Im ersteren Fall würde man folgerichtig eine adäquate antipsychotische Behandlung empfehlen und hätte damit möglicherweise zu einer Verkürzung der „Duration of Untreated Psychosis (DUP)“ in der betreffenden Region beigetragen. Dass derartige DUP-Verkürzungen sinnvoll wären, weil Patienten mit längerer DUP signifikant schlechtere Behandlungsund psychosoziale Wiedereingliederungsergebnisse aufweisen, kann man nach dem hierfür inzwischen erreichten hochrangigen Evidenzgrad nicht mehr gut in Zweifel ziehen [4]. Danach sollte sicherlich ,jeder Schizophrene“ so früh wie möglich nach den für Erstepisoden geltenden Leitlinien behandelt werden.

In den genannten Risikozuständen dagegen sind definitionsgemäß noch keine oder noch keine voll ausgeprägten psychotischen Symptome zu erkennen, sodass sich auch psychotische Störungen noch gar nicht diagnostizieren und die Betroffenen erst recht noch nicht als „Schizophrene“ einstufen lassen. Eine Erfüllung der sog. „Ultra-High-Risk(UHR)“-Kriterien würde nach den bislang vier diesbezüglich aussagekräftigen Früherkennungsstudien bedeuten, dass bei durchschnittlich knapp 40\% der Betroffenen bereits innerhalb der nächsten zwölf Monate 
mit dem Ausbruch einer ersten psychotischen Episode zu rechnen wäre. In den ebenfalls vier bisher durchgeführten prospektiven, randomisierten und kontrollierten Studien zur indizierten Prävention auf dieser Risikostufe kamen kognitive Verhaltenstherapie (KVT) im Einzelverfahren zusammen mit niedrig dosiertem Risperidon [5], Olanzapin in Kombination mit supportivpsychoedukativer Einzel- und Familienintervention [6], niedrig dosiertes Amisulprid zusammen mit supportiver psychosozialer Intervention [7] sowie einmal auch Einzel-KVT allein [8] zum Einsatz. Zweimal ließ sich in der Tat die Inzidenz erster psychotischer Episoden signifikant reduzieren, einmal verblieb dieser Präventionseffekt auf Trendniveau und die jüngste dieser vier Studien zeigt zwar schon signifikante Verbesserungen der Symptome und der sozialen Anpassung, lässt aber noch keine abschließende Beurteilung hinsichtlich möglicher Präventionseffekte zu.

Der Nachweis der psychosefernen Risikostufe liefe darauf hinaus, dass innerhalb der nächsten 2 - 3 Jahre etwa 50\% der Betroffenen eine erste psychotische Episode entwickeln [3]. Diese Aussagen zur Prädiktionskraft stützen sich bisher nur auf zwei Früherkennungsstudien, von denen die eine allerdings einen durchschnittlich knapp 10-jährigen Verlaufszeitraum überschaute und dementsprechend besonders aussagekräftig war [9]. Umso interessanter und vielversprechender erscheint es jetzt, dass die gerade zum Abschluss gelangende erste hierauf bezogene prospektive, randomisierte und kontrollierte Frühinterventionsstudie rein mit psychologischen Mitteln überzeugende Präventionseffekte erzielen konnte. In der Gruppe, die an einem auf KVT-Basis neu entwickelten multimodalem Programm teilgenommen hatte, sind weniger Übergänge in die psychosennähere Risikostufe erfolgt und vor allem auch signifikant weniger schizophrenieforme und schizophrene Störungen aufgetreten [10].

Insgesamt befindet sich bei dieser Studienlage die indizierte Prävention schizophrener Störungen sicherlich noch im Stadium der wissenschaftlichen Erprobung. Wenn die Entwicklung auf diesem innovativen Gebiet aber weiter so voranschreitet wie bisher, ließen sich schon in den nächsten Jahren evidenzbasierte Ergebnisse in die Versorgungspraxis umsetzen und möglichst jedem Ratsuchenden mit Frühwarnzeichen auf die individuellen Bedürfnisse zugeschnittene Präventionsangebote unterbreiten.

Univ.-Prof. Dr. Joachim Klosterkötter

Klinik und Poliklinik

für Psychiatrie und Psychotherapie

Universität zu Köln

Kerpener Straße 62

50924 Köln

E-mail: joachim.klosterkoetter@uk-koeln.de

\section{Kontra}

\section{Ronald Bottlender}

Die vielfach gefundene Assoziation zwischen längerer DUP (Duration of Untreated Psychosis) und ungünstigerem Outcome $[11,12]$ ist eine der Hauptstützen für die Hypothese, dass über eine Verkürzung der DUP, eine Verbesserung des Krankheitsver- laufs der Schizophrenie erreicht werden kann. Darüber hinaus wird derzeit der Ansatz verfolgt, „Patienten“ sogar schon vor Ausbruch der ersten Psychose zu identifizieren und zu behandeln. Hierdurch soll der Ausbruch der Schizophrenie verhindert oder wenigstens verzögert und deren Langzeitverlauf verbessert werden.

Was spricht nun gegen dieses Konzept? Prinzipiell ist hier festzuhalten, dass therapeutisches Handeln stets am Einzelfall ausgerichtet ist. Allaussagen im Sinne von „Jeder Patient wird so und so behandelt" sind insofern für die Medizin wenig geeignet. Die Eingangsthese verkennt ferner, dass das primäre Problem in der Schizophreniebehandlung oft nicht im Zeitpunkt, sondern in der Akzeptanz und Compliance der Patienten liegt. Bis zu 89\% der schizophrenen Patienten sind bezüglich ihrer Behandlung incompliant [13]. Solange aber diese Problematik nicht nachhaltig angegangen wird, steht zu befürchten, dass auch eine frühe und dann eben oft von Incompliance gefolgte Therapie den Verlauf der Schizophrenie nicht substanziell ändern wird. Abgesehen von diesen Punkten lassen sich aber noch spezifischere Punkte gegen die o.g. Devise ins Feld führen.

Die Evidenzen bezüglich der Assoziation zwischen DUP und Outcome sind zwar zahlreich und recht konsistent, dennoch handelt es sich hierbei zunächst einmal nur um korrelative Zusammenhänge. Korrelationen sind aber per se noch keine Kausalzusammenhänge. So bedeutet etwa der statistische Zusammenhang zwischen längeren Haaren und erhöhtem Brustkrebsrisiko weder, dass längere Haare karzinogen sind, noch dass durch eine Verkürzung der Haarlänge das Krebsrisiko gesenkt wird. Missing Link dieser Assoziation ist das Geschlecht. Ähnlich könnte es sich bezüglich des Zusammenhangs zwischen DUP und schizophrenem Krankheitsverlauf verhalten. Empirische Daten, die prospektiv belegen, dass eine experimentelle Reduktion der DUP tatsächlich eine Verbesserung des schizophrenen Krankheitsverlaufs nach sich zieht, fehlen bis dato.

Nun könnte man aber einwenden, dass eine Frühtherapie schon allein deswegen sinnvoll ist, weil dadurch Leiden verkürzt und größerer Schaden verhindert wird. Diesem Standpunkt ist zu entgegnen, dass frühe Therapie durchaus schädlich sein kann, wie dies u.a. am Beispiel des Debriefings zur Prävention der PTSD eindrucksvoll belegt wurde [14]. Folge einer Frühtherapie bei Schizophrenie könnte sein, dass vermehrt auch solche Patienten frühzeitig den Risiken einer Behandlung ausgesetzt werden, deren Psychose - hätte man den Spontanverlauf abgewartet - auch ohne spezifische und insbesondere kontinuierliche Therapiemaßnahmen abgeklungen wäre. Die Größenordnung dieser Gruppe ist durchaus relevant und dürfte mit jener der Gruppe von 1-Episoden-Verläufen, welche studienabhängig bei bis zu einem Drittel liegt [15], vergleichbar sein. Berücksichtigt man ferner, dass bei generell früh einsetzender Therapie - und dies ist eine immanente Tendenz der Früherkennungsprogrammatik nicht nur schizophrene Patienten, sondern z.B. auch Patienten mit zu Spontanremissionen neigenden vorübergehenden psychotischen Störungen von der Frühtherapie betroffen wären, stiege dieser Prozentsatz weiter an.

Die Frage, die sich hieraus ergibt, lautet: Wie lässt sich also die Therapie von Patienten, die auch ohne Therapie einen günstigen 
Verlauf gehabt hätten, vermeiden? Wie zuvor dargestellt, ist dieses Problem schon bei manifest Erkrankten in bedeutsamem Ausmaß vorhanden, noch gravierender wird es aber, wenn die Frühtherapie Personen betrifft, deren Psychose noch gar nicht manifest ist, und bei denen lediglich ein Psychoserisiko postuliert wird.

Die mit heutigen Methoden identifizierbaren Risikopersonen entwickeln innerhalb eines Jahres zwischen 20 und 60\% erstmalig das Vollbild einer Psychose [16]. Vice versa heißt dies aber, dass zwischen 40 und $80 \%$ dieser Risikopersonen keine Psychose entwickeln. Würde man nun bei Risikopersonen generell eine Therapie empfehlen, bedeutete dies, dass die Therapie in bis zu $80 \%$ der Fälle prinzipiell nicht indiziert wäre. Klosterkötter et al. [9] berichteten zwar vergleichsweise bessere Prädiktionswerte mit einer falsch Positivenrate von unter 10\%. Da in ihrer Studie die hoch prädiktiven Symptome anfänglich aber nur bei etwa einem Drittel der Patienten vorlagen und die Rate an falsch Negativen mittels dieser Symptome ähnlich hoch lag, ist eine breitere Früherkennung auch mit dieser Methodik nicht praktikabel. Dies gilt umso mehr, wenn man berücksichtigt, dass diese Daten an speziellen Risikogruppen erhoben wurden und somit nicht ohne weiteres auf die Allgemeinbevölkerung, die Ziel einer breit angelegten Präventionsstrategie wäre, übertragbar sind. Epidemiologische Studien zeigen Prävalenzen psychotischer Symptome in der Allgemeinbevölkerung, die bis zu 50fach höher liegen als die der Schizophrenie [17]. Bei einem Screening der Allgemeinbevölkerung ist also zu erwarten, dass die Rate falsch Positiver rapide ansteigt. Auch würde die von Klosterkötter berichtete positive prädiktive Bedeutung ihres Instrumentes bei Anwendung in der Allgemeinbevölkerung von 70 auf lediglich $2 \%$ abfallen [18].

Angesichts dieser Problematik wird von den Protagonisten der Früherkennung nun überwiegend die indizierte Prävention propagiert, bei der nur solche Personen gescreent und behandelt werden, die quasi aus eigenen Bedürfnissen heraus ein Früherkennungszentrum aufsuchen. Dieser Legitimationsversuch löst das Problem aber nur scheinbar. Auch mit dieser Strategie werden weiterhin zahlreiche Personen, die in ihrem Leben nie eine Psychose entwickelt hätten, unnötigerweise der Information über ein bestehendes Psychoserisiko wie auch dem Risiko von Nebenwirkungen und sozialer Stigmatisierung ausgesetzt.

Die Früherkennung und Therapie der Schizophrenie ist ein neuer und faszinierender Ansatz. Allem Enthusiasmus zum Trotz darf gleichwohl nicht übersehen werden, dass auf diesem Gebiet weiterhin mehr Fragen offen als beantwortet sind. Nutzen und Schaden der Früherkennung bedürfen weiterer sorgfältiger Abwägung. Unabhängig davon, kann die Frühtherapie der Schizophrenie langfristig nur dann nachhaltig sein, wenn auch bereits bestehende Therapieangebote z. B. im Sinne integrierter Theapieansätze optimiert werden [19].

PD Dr. med. habil. Ronald Bottlender

East London and the City Mental Health NHS Trust

Department of Psychiatry

Newham Centre for Mental Health

Glen Road, Cherry Tree Way, London E13 8SP

E-mail: Ronald.Bottlender@elcmht.nhs.uk

\section{Literatur}

${ }^{1}$ WHO. Prevention of Mental Disorders. Effective Interventions and Policy Options. Summary Report. Geneva: World Health Organization, 2005. http://www.who.int/mental_health/evidence/en/Prevention_ of_Mental_Disorders.pdf

2 Philipps LJ, McGorry PD, Yung AR, McGlashan TH, Cornblatt B, Klosterkötter J. Prepsychotic phase of schizophrenia and related disorders: recent progress and future opportunities. Br J Psychiatry 2005; 48, suppl: $33-44$

${ }^{3}$ Ruhrmann S, Schultze-Lutter F, Klosterkötter J. Early detection and intervention in the initial prodromal phase of schizophrenia. Pharmacopsychiatry 2003; suppl 3: $162-167$

${ }^{4}$ Marshall M, Lewis S, Drake R, Jones P, Croudace T. Association between duration of untreated psychosis and outcome in cohorts of first-episode patients. Arch Gen Psychiatry 2005; 62: 975-983

${ }^{5}$ McGorry PD, Yung AR, Philipps LJ, Yuen HP, Francey S, Cosgrave EM, Germano D, Bravin J, McDonald T, Blair A, Adlard S, Jackson H. Randomized controlled trial of interventions designed to reduce the risk of progression to first-episode psychosis in a clinical sample with subthreshold symptoms. Arch Gen Psychiatry 2002; 59: 921 - 928

${ }^{6}$ McGlashan TH, Zipursky RB, Perkins DO, Addington J, Woods SW, Miller TJ, Lindborg S, Marquez E, Hawkins K, Hoffmann RE, Tohen M, Breier A. The PRIME North America randomized double-blind clinical trial of olanzapine versus placebo in patients at risk of being prodromally symptomatic for psychosis: I. study rational and design. Schizophr Res 2003; 61: 7-18

${ }^{7}$ Bechdolf A, Ruhrmann S, Wagner M, Kuhn KU, Janssen B, Bottlender R, Wieneke A, Schultze-Lutter F, Maier W, Klosterkötter J. Interventions in the initial prodromal states of psychosis in Germany: Concept and recruitment. Br J Psychiatry 2005; 48, suppl: 45-48

${ }^{8}$ Morrison AP, French P, Walford L, Lewis SW, Kilcommons A, Green J, Parker S, Bentall RP. A randomised controlled trial of early detection and cognitive therapy for the prevention of psychosis in people at ultra-high risk. Br J Psychiatry 2004; 185: 291 - 297

${ }^{9}$ Klosterkötter J, Hellmich M, Steinmeyer EM, Schultze-Lutter F. Diagnosing schizophrenia in the initial prodromal phase. Arch Gen Psychiatry $2001 ; 58$ : $158-164$

${ }^{10}$ Bechdolf A, Veith V, Klosterkötter J. Group therapy for people at high risk of developing psychosis. In: Addington, Francey, Morrison (eds): Working with people a high risk of developing psychosis: A treatment handbook. Chichester: John Wiley \& Sons, Ltd., 2006

${ }^{11}$ Bottlender R, Möller H-J. The impact of the duration of untreated psychosis on short- and long-term outcome in schizophrenia. Current Opinion in Psychiatry 2003; 16, Supplement 2: 39-43

${ }^{12}$ Marshall M, Lewis S, Lockwood A, Drake R, Jones P, Croudace T. Association between duration of untreated psychosis and outcome in cohorts of first-episode patients: a systematic review. Arch Gen Psychiatry 2005; 62 (9): 975-983

${ }^{13}$ Gilmer TP, Dolder CR, Lacro JP, Folsom DP, Lindamer L, Garcia P, Jeste DV. Adherence to treatment with antipsychotic medication and health care costs among Medicaid beneficiaries with schizophrenia. Am J Psychiatry 2004; 161 (4): $692-699$

${ }^{14}$ Rose S, Bisson J, Wessely S. A systematic review of single-session psychological interventions („debriefing“) following trauma. Review. Psychother Psychosom 2003; 72 (4): 176-184

${ }^{15}$ Os J van, Wright P, Murray R. Follow-up studies of schizophrenia I: snatural history and non-psychopathological predictors of outcome. European Psychiatry 1997; 12, Supplement 5: 327s-341s

${ }^{16}$ McGlashan TH, Miller TJ, Woods SW. Pre-onset detection and intervention research in schizophrenia psychoses: current estimates of benefit and risk. Schizophr Bull 2001; 27 (4): 563-570

17 Os J van, Hanssen M, Bijl RV, Vollebergh W. Prevalence of psychotic disorder and community level of psychotic symptoms: an urban-rura comparison. Arch Gen Psychiatry 2001; 58 (7): 663-668

${ }^{18}$ Warner R. Early intervention in schizophrenia: a critique. Epidemiologia e Psichiatria Sociale 2002; 11, 4: 248-255

${ }^{19}$ Petersen L, Jeppesen P, Thorup A, Abel MB, Ohlenschlaeger J, Christensen TO, Krarup G, Jorgensen P, Nordentoft M. A randomised multicentre trial of integrated versus standard treatment for patients with a first episode of psychotic illness. BMJ 2005; 331 (7517): 602 -605 